\title{
Espiritualidad y educación en la sociedad del conocimiento
}

Margott Piedra Hernández

Profesora de apoyo del Programa de Licenciatura en Docencia, Escuela de Ciencias de la Educación, UNED, Costa Rica.

mpiedrah@uned.ac.cr

Recibido: 12 de octubre del 2017

Corregido: 21 de noviembre del 2017

Aceptado: 24 de noviembre del 2017

\section{Resumen}

La espiritualidad está envuelta en confusión y malos entendidos principalmente al asociarse a la religión, sin embargo, no constituye un sistema de creencias y dogmas acerca del bien o el mal, se trata de una construcción multidireccional y permanente para alcanzar la comprensión de sí mismo, el mundo, la naturaleza y el cosmos.

A partir de este reconocimiento el ensayo busca demostrar el papel de la educación en el desarrollo y fortalecimiento de la espiritualidad; para ello, se muestran dos perspectivas teóricas que intentan explicar la espiritualidad más allá de la religión: por un lado, aquella sustentada en la neurociencia que vincula la espiritualidad a las capacidades del cerebro, por otro, la perspectiva holística que retoma el concepto de integralidad y totalidad, recalcando la importancia de la dimensión espiritual para el desarrollo pleno de la condición humana.

Se comprueba que ambas posiciones parecen coincidir en la necesidad de potenciar la espiritualidad para superar y transformar las limitaciones de la sociedad en la que vivimos, donde lo material y la satisfacción de las necesidades biológico-corporales se asumen como único medio para alcanzar la felicidad.

Finalmente, se plantea cómo para alcanzar el equilibrio entre las dimensiones constituyentes del ser humano, las instituciones educativas deben asumir su responsabilidad y compromiso, a través de prácticas espirituales que desde el planeamiento de las actividades cotidianas, los programas de estudio y el currículo enseñen a vivir, propiciando el autodescubrimiento y el reconocimiento-fortalecimiento de valores que dan sentido a la existencia.

Palabras clave: Sociedad del conocimiento, neurociencia, educación integral, holismo, espiritualidad.

\section{Abstract \\ Spirituality and education in the knowledge society}

Spirituality is involved in confusion and misunderstanding mainly by associating with religion, however, spirituality does not constitute a belief system and dogmas about good or evil, it is a multidirectional and permanent construction to achieve self-understanding. same, the world, nature and the cosmos. 
From this recognition this essay seeks to demonstrate the role of education in the development of spirituality, for it, two theoretical perspectives that try to explain spirituality beyond religion are shown: on the one hand, those sustained in neuroscience that connect spirituality to the capacities of the brain, and on the other, the holistic perspective that takes up the concept of integrality and totality, emphasizing the importance of the spiritual dimension for the full development of the human condition.

It is verified that both positions seem to coincide in the need to enhance spirituality to overcome and transform the limitations of the society in which we live, where the material and the satisfaction of biological-corporal needs are assumed as the only means to achieve happiness.

Finally, it is proposed how to reach the balance between the constituent dimensions of the human being, the educational institutions must assume their responsibility and commitment, through spiritual practices that from the planning of the daily activities, the study programs and the curriculum teach to live, fostering self-discovery and recognition-strengthening values that give meaning to existence.

Key words: Society of knowledge, neuroscience, education, holism, spirituality.

\section{INTRODUCCIÓN}

El mundo experimenta desde mediados del siglo pasado un cambio en las fuentes tradicionales de poder: el capital, el trabajo y la tierra han dado lugar a una inagotable nueva fuerza como es el conocimiento. La sociedad del conocimiento supone un cambio en la forma cómo las sociedades se construyen y reconstruyen a sí mismas, al respecto Tedesco citado por Aguerrondo (s.f.) menciona:

[...] el conocimiento tiene virtudes intrínsecamente democráticas. A diferencia de las fuentes de poder tradicionales (la fuerza, el dinero, la tierra) el conocimiento es infinitamente ampliable. Su utilización no lo desgasta sino que, al contrario, puede producir más conocimiento. Un mismo conocimiento, puede ser utilizado por muchas personas y su producción exige creatividad, libertad de circulación, intercambios, críticas constructivas, diálogo. Todas ellas condiciones propias de una sociedad democrática. (p. 1)

La realidad obliga a una revolución en la función de la educación en la sociedad, que exige una transformación, un nuevo paradigma, en el que no basta con la introducción de nuevos y más complejos "saberes"; bajo el nuevo paradigma la actitud pasiva y contemplativa ante la realidad deja de existir, ya no se trata de reproducir sino de crear, de de hacer.

Pero la transformación debe venir de la mano de un compromiso ético, construyendo una escala de valores acordes con las necesidades planetarias, se trata de una práctica educativa sustentada desde la espiritualidad como esperanza del y para el cambio, que enseñe a vivir y propicie el autodescubrimiento, el reconocimiento y el fortalecimiento de valores que dan sentido a la existencia.

En el ensayo se discute acerca de la necesidad de la espiritualidad en el nuevo paradigma educativo, para el reconocimiento y atención al "ser" respetando la multidimensionalidad e integralidad de la persona, principios sobre los cuales sus dimensiones constituyentes actúan en íntima relación para el desarrollo de la interioridad, el sentido de la vida, la realización individual y la dignidad.

Así, el quehacer educativo debe atender el "ser" por medio de prácticas desarrolladas desde el cuidado, la solidaridad, el diálogo, el amor, propiciando y respetando el pensamiento divergente, la creatividad, 
la convivencia en respeto y responsabilidad; ya que sin los principios señalados, el aprendizaje se reduce a una mecánica sin significado humano, al servicio de la formación para el trabajo y el rendimiento.

\section{DEFINIENDO LA ESPIRITUALIDAD}

La espiritualidad, contraria a las otras dimensiones constituyentes del individuo está envuelta en un velo de confusión y malos entendidos, en parte porque no se ha enseñado a entenderla ni fortalecerla, como consecuencia es confundida o asociada con la metafísica y muy especialmente con las confesiones religiosas. En el primer caso, la confusión procede del carácter que ha tomado esta rama de la filosofía en las últimas décadas, especialmente al asociarse con el esoterismo.

El segundo caso es mucho más complejo, principalmente porque las referencias a la espiritualidad vienen la mayoría de las veces de los discursos eclesiásticos:

La expresión "valores espirituales" aparece con más frecuencia en los labios de los sacerdotes y obispos de tendencia conservadora. De ellos es común escuchar que la crisis del mundo contemporáneo se encuentra fundamentalmente en el abandono del mundo espiritual: la no asistencia a misa o cualquier otra referencia explícita a la Iglesia jerárquica. (Boff, 2013, párr. 4)

Las religiones pueden explorar el mundo espiritual y así ser coherentes con sus propios principios, de hecho se puede afirmar que no existen sin espiritualidad, sin embargo no es lo mismo religión que espiritualidad, porque el objetivo primordial de las religiones es crear sistemas de interpretación de las concepciones, los valores, los cuales son inmutables dado que están fundamentados en el sentido de revelación divina que es intocable e inalterable, "[...] el término sugiere fijación, dominio, sumisión, control del pensamiento y del sentir, control de la moralidad y de los modos de vida, de las creencias. "Religión" evoca ideas de exclusivismo y exclusión" (Corbí, 2007, pp. 151-152).

Por el contrario, la espiritualidad no constituye un sistema de creencias acerca del bien o el mal, es decir que la espiritualidad puede existir sin la religión. De hecho se podría afirmar que ha surgido un nuevo interés por el tema desvinculado de la religión, a partir de esta nueva forma "anti-sistémica" de entenderla aparecen dos grandes tendencias en los estudios sobre el tema: un primer grupo que vincula la espiritualidad con las capacidades de la mente, lo que al fin de cuentas la encadena a la esfera biológica-corporal del ser humano, en esa línea encontramos, entre otros, al Doctor Howard Gardner, quien se refirió en su teoría de las inteligencias múltiples (1983), a un tipo de inteligencia a la que denominó: "inteligencia existencial o transcendente", la cual se manifiesta en la necesidad de planearse y preocuparse por los aspectos espirituales, evidenciado en cuestionamientos sobre el significado de la vida, la muerte o el amor.

Igualmente, los doctores Danah Zohar e lan Marshall (1997) van más allá al descubrir que las prácticas espirituales generan oscilaciones en las ondas electromagnéticas que se reproducen sobre las neuronas, vinculando el concepto de "espiritualidad" con el de "inteligencia", al tiempo que insisten en demostrar que la inteligencia espiritual es la inteligencia primordial pues constituye la base fundamental para el efectivo funcionamiento del cociente intelectual.

Dentro del ámbito de la genética, Dean Hamern (2004), demostró mediante la comparación de muestras de ADN de más de 1000 personas de diferentes edades y contextos sociales, que el ser humano posee un gen especializado que hace posible el desarrollo de la dimensión y la actividad espiritual. El gen fue llamado VMAT2 "gen de la espiritualidad" abriendo así la posibilidad científica de la actividad espiritual. 
Bajo esos mismos principios el neurocientífico Francisco Rubia (2015), a través de numerosas investigaciones confirmó que el cerebro genera experiencias espirituales o de trascendencia. Experiencias que se pueden hiperactivar mediante la estimulación de las estructuras cerebrales del sistema límbico también llamado cerebro emocional; a partir de los hallazgos se ha creado un nuevo concepto: la neuroespiritualidad, reconociendo así que las personas tienen una disposición innata a la espiritualidad.

Finalmente, en la primera tendencia el sistema límbico adquiere especial importancia para quienes encuentran las explicaciones de la espiritualidad en las capacidades infinitas del cerebro humano, ya que en esta área se localizan las amígdalas, las cuales constituyen centros de referencia para la percepción de los estímulos relacionados con las emociones, mismas que están ligadas al sentido de trascendencia "[...] lo que de forma genérica se llama trascendencia constituye un elemento central de la experiencia mental humana" (Nogués, 2012, p. 54).

La segunda posición en las investigaciones sobre la espiritualidad que se mencionó anteriormente, retoman el concepto de integralidad y totalidad, recalcando su importancia para el desarrollo pleno de la condición humana, al respecto Nogués (2012) afirma que la vida mental no se refiere únicamente al cerebro como un complejo sistema que abarca todo el organismo "[...] No existen, pues, funcionamientos mentales que puedan referirse de forma totalmente exclusiva al cerebro. Toda pasión, idea, actitud, emoción, razonamiento [...] son vividos como acción conjunta de todo el organismo" (p. 35).

En esa dirección se encuentra Abraham Maslow, (1943) en su pirámide o jerarquía de las necesidades humanas, aportó el término "autorrealización", la cual consiste en un estado espiritual en el que el individuo emana creatividad, es feliz, tolerante, posee un propósito y una misión de ayudar a los demás a alcanzar el estado de sabiduría y beatitud.

La importancia de atender la espiritualidad desde la comprensión de la integralidad humana constituye la base de las ideas de Viktor Frankl, para quien lo espiritual es una fuerza, no una substancia, de esa manera la espiritualidad de la persona no es sólo una característica -igual que lo son lo corporal y lo psíquico-, sino un "constituens", algo que distingue al ser humano (Frankl, 1990, p. 78).

La existencia espiritual está 'soldada" en la existencia humana, una separación clara entre ambas sólo se puede hacer desde un punto de vista heurístico. Según el objetivo de la reflexión sobre el hombre se abordará a éste más desde la unidad-totalidad o desde la fragmentación en lo espiritual y lo psicofísico. No obstante, una perspectiva no puede olvidar la existencia de la otra. (Noblejas, 1994, p. 47)

Tal como afirmó Pierre Teilhard (religioso y filósofo francés de finales del siglo XIX y principios del XX) somos seres espirituales teniendo una experiencia humana. Bajo estos principios se propone que la espiritualidad no es una parte del ser humano, representa su "totalidad vital" (Boff, 1992, p.37), y se expresa en las distintas formas del vivir de cada ser humano; esto invita a entender que la espiritualidad no es unidireccional, intangible, única, inmutable, creada, subjetiva, inequívoca, silente; por el contrario, constituye una construcción multidireccional, permanente en cada día, interna y externa, teoría y praxis, dialogante y silente al mismo tiempo, es un estado amoroso por todo y por todos. Consiste en la comprensión interiorizada de sí mismo, el mundo, la naturaleza y el cosmos.

\section{LO ESPIRITUAL EN LA SOCIEDAD DEL CONOCIMIENTO}

Pese a los intentos por demostrar no solo la existencia, sino la necesidad, el reconocimiento y el respeto a la integralidad vital de la condición humana, la nueva sociedad lleva en su naturaleza una fragmentación como nunca en la historia del pensamiento humano, en áreas del saber, magnificando la tecnología 
como solución a todos los problemas y reasignando disciplinas tales como el arte, la historia, la filosofía a espacios de ocio, llevando a un desequilibrio ontológico que ha originado una crisis de paradigmas y valores, lo cual incapacita a las personas para entender las complejidades de la realidad.

[...] la supremacía del conocimiento fragmentado según las disciplinas impide a menudo operar el vínculo entre las partes y las totalidades y debe dar paso a un modo de conocimiento capaz de aprehender los objetos en sus contextos, sus complejidades, sus conjuntos. (Morin, 1999, p. 2)

En la situación descrita radica la incoherencia de la sociedad del conocimiento: un mundo y una naturaleza humana que no son articulados desde su complejidad, ya que no solo se ha desarticulado el conocimiento, sino muy especialmente lo humano, lo cual conduce a un debilitamiento de la percepción global del conocimiento, de la responsabilidad individual, social, planetaria y particularmente del "ser".

Puesto que lo humano se entiende también como fragmentado, se ha desarrollado una profunda contradicción: las particularidades de lo material asumen mayor relevancia frente a aquellas cualidades inmateriales, de ese modo, la persona se concibe y se construye fundamentalmente desde su dimensión biológica desarticulando sus dimensiones espirituales, psicológicas y sociales.

El resultado es una sociedad en la que se dedican grandes esfuerzos a cultivar la dimensión biológica-material, en ese sentido, la familia y otras instituciones sociales como la escuela perpetúan prácticas que refuerzan la idea de desarrollarse única y plenamente en esa dimensión para encontrar la felicidad; bajo esos principios, cada individuo se construye a sí mismo para formar parte de una masa estandarizada, en la que por consecuencia termina por sentirse alienado y deshumanizado.

Otra amenaza ha sido el crecimiento de una perspectiva cultural que está determinando una manera de ser idólatra y fragmentada que trae limitantes en su propia expresión. En otras palabras, el ser humano parece preferir limitarse a su propia autoconcepto y eso lo lleva a un apego acrítico a instituciones y modelos que dan estabilidad. (Garcez, 2014, p. 9)

La discusión acerca de la sociedad del conocimiento y su papel en la disgregación de la integralidad humana, recuerda que si bien la materia es uno de los componentes de la realidad, no lo es todo, ni siquiera para la ciencia, pues junto a la masa se debe percibir la energía. Al igual que ocurre entre la relación masa-energía, el ser humano constituye una totalidad una unidad substancial entre sus dimensiones psicológica-física-espiritual, pero es sólo por medio del reconocimiento de su espiritualidad "[...] que el hombre penetra en la estructura más profunda de la realidad" (Lugo y Barni, 2014, p.33).

De ese modo, el materialismo fragmenta no solo el conocimiento sino a la persona y se convierte en la negación del pensamiento, de la filosofía, la cultura y de la vida, pues se trata de un tipo de saber en función del interés del "yo", por tanto es limitado.

Como tal ese conocimiento, mientras tenga la función que tiene, no puede trascender esa su naturaleza, y como otro Rey Midas, todo lo que toca, valga decir todo lo que sobre él se construye se convierte necesariamente en instrumental y objeto, producto egocentrado e interesado. (Robles, 2011, p. 46)

El modelo de sociedad que se ha construido, conlleva por sí mismo desafíos para los cuales el ser humano no se halla preparado, derivando en un mundo en crisis, en el que se fortalecen actitudes como el consumismo, el egoísmo, la codicia, el irrespeto, el desamor y en general la falta de cuidado por toda forma de vida, la destrucción planetaria, la desigualdad y la pobreza, las altas tasas de suicidios y homicidios son algunas evidencias de ello. 
Así, el siglo XX ha vivido bajo el reino de una seudo-racionalidad que ha presumido ser la única, pero que ha atrofiado la comprensión, la reflexión y la visión a largo plazo. Su insuficiencia para tratar los problemas más graves ha constituido uno de los problemas más graves para la humanidad. (Morín, 1999, p. 20)

En el contexto de esta visión reduccionista de la realidad, en la que se menosprecia todo aquel conocimiento que no sea técnico y "funcional", la educación ha sido la encargada de desarticular más y más esa complejidad desde currículos fragmentados en disciplinas. Es a lo que Edgar Morín (2009) llama inteligencia ciega, que imposibilita ver la totalidad fragmenta:

[...] destruye desde el óvulo las posibilidades de comprensión y de reflexión; reduce las oportunidades de un juicio correctivo o de una visión a largo plazo... Incapaz de proyectar el contexto y el complejo planetario, la inteligencia ciega se vuelve inconsciente e irresponsable. (p. 30)

Las limitaciones de la sociedad en la que vivimos, obliga a concluir que el ser humano sin cualquiera de sus dimensiones está incompleto, pero sin su dimensión espiritual carece de aquello que más debe caracterizarlo, Viktor Frank (2002) lo reafirma al comprender que la espiritualidad otorga dignidad a la persona, entendiéndose a sí misma como algo más que un elemento anónimo de la naturaleza y la sociedad.

Autores como Robles (2014) y Corbí (2007) insisten en la importancia de esa dimensión al llamarla "dimensión o cualidad humana absoluta", estas condiciones obligan a las sociedades del siglo XXI a tratar de encontrar el equilibrio. La apuesta se encuentra en la educación, se trata de una actividad educativa orientada hacia la trascendencia y el descubrimiento de un sentido de vida más profundo e integrador de todas las dimensiones humanas.

De esa forma se reconocerá y atenderá al "ser" respetando la multidimensionalidad e integralidad de la persona, principios sobre los cuales sus dimensiones actúan en íntima relación para el desarrollo de su exterioridad e interioridad, así como para lograr el sentido de la vida, la realización individual y la dignidad.

Nosotros los seres humanos somos una unidad compleja, cuerpo-espíritu. Somos cuerpo y espíritu a la vez, portadores de exterioridad y de interioridad. Necesitamos comer, beber, relacionarnos, celebrar, vivir y convivir. Pero también necesitamos establecer una relación interiorizada para absorber la resonancia de lo exterior y hacer nuestras propias síntesis. Elaborar, en la intimidad de nosotros mismos, nuestros deseos, sentimientos, dudas y pensamientos. (Leme, 2015, p. 50)

Es un error pensar que una educación secular o laica no debe ocuparse de la la integralidad de la condición humana, pues la espiritualidad como se ha demostrado concierne a lo antropológico y no únicamente a la fe; no obstante, con la distancia cada vez mayor de las sociedades con respecto a las experiencias religiosas, se están anulando las únicas instancias que se han ocupado de esa dimensión.

La importancia de la espiritualidad en las prácticas educativas radica en la referencia al ser esencial-interior, de manera que con su desarrollo se logra ampliar el sentido del "ser", la capacidad de discernir serenamente, lo cual conduce al interés incondicional por todos y por todo, al amor y a la paz; es decir, bajo estos principios, se cultivará una transformación profunda que alcance no sólo las relaciones intra e interhumanas, sino también con la naturaleza.

Sin los principios expuestos, se reduce el aprendizaje a una mecánica sin significado humano, al servicio de la formación para el trabajo y el rendimiento; por el contrario, las prácticas educativas cultivadas desde 
la espiritualidad serán desarrolladas desde el cuidado, la solidaridad, el diálogo, el amor, propiciando y respetando el pensamiento divergente, la creatividad, la convivencia en respeto y responsabilidad.

Pero no se trata de un cuidado como conducta externa del individuo, se trata en palabras de Boff (2008) "[...] que el cuidado posee una dimensión ontológica que entra en la constitución del ser humano. En un modo-de-ser singular del hombre y de la mujer. Sin cuidado dejamos de ser humanos" (p.89). Se asiste, como se mencionó anteriormente, a una espiritualidad de acción y no de contemplación, en la que el docente en concordancia con el respeto y desarrollo de su propia dimensión espiritual deberá desarrollar en su quehacer educativo los principios de una educación más humana y relacional, que responda a las urgencias planetarias e individuales.

\section{LO ESPIRITUAL, ESENCIA DE LA EDUCACIÓN}

La espiritualidad en la educación se configura en la realización personal y vocacional, que procede del amor por lo que se hace y por quienes se hace, así como de la esperanza del poder de la educación para transformar,

[...] la realización personal no es obra de la razón que discurre de aquí para allá, sino del recogimiento del espíritu que capta la riqueza de cada situación [...] El espíritu es el modo de ser del hombre que sabe descubrir el sentido de cada cosa. (Boff, 1992, 37)

El respeto y el desarrollo de lo espiritual debe convertirse en un compromiso indefectible de la educación; compromiso alcanzado mediante prácticas educativas que, desde el planeamiento de las actividades cotidianas, los programas de estudio, el currículo, la participación de comunidad educativa y comunitaria, conviertan en realidad y no sólo en intención a lo espiritual como esencia de la educación.

No obstante, los modelos educativos tradicionales sustentados en corrientes filosóficas y epistemológicas tales como el pragmatismo, el conductismo o el reduccionismo continúan desarrollando currículos que prescinden de la dimensión espiritual o peor aún la presuponen. Al respecto Maya (2016) insiste en recordar la necesidad de cambio en los paradigmas que ya han cumplido su misión:

Sorprende que servicios y actividades como la educación transiten aún en viejas y anacrónicas propuestas en la persistencia de seguir educando para un mundo, para una realidad social, económica, política, ambiental, cultural y axiológica que ya no es, porque aunque parezca insólito paradójico, la educación es uno de los sectores del conocimiento y de la actividad humana donde al parecer se registra con frecuencia mayor resistencia al cambio y, por ende, en términos de los mismo se padece mayor parálisis paradigmática. (pp. 24-25)

Bajo los enfoques llamados tradicionales el ser humano se convierte en un ser desestructurado, estático, alienado al servicio de un sistema socioeconómico del trabajo y la eficiencia; olvidando en sus prácticas la identidad compleja del ser humano: como ser físico, pero también psíquico, cultural, social, histórico y espiritual. El Ministerio de Educación de Costa Rica así lo establece en su política Educativa hacia el Siglo $\mathrm{XXI}$ al reconocer:

La visión integral del ser humano sólo puede llevarse a la práctica mediante una oferta educativa en la que los conocimientos, los procesos para construirlos y reconstruirlos, y la aplicación de esos conocimientos en el desarrollo de la persona y de la sociedad se encuentran incorporados en el proceso educativo sin detrimento alguno. (MEP, 1994, p.1) 
En la práctica del aula, lo anterior se traduce en una labor docente que favorece la generación de ambientes libres del materialismo y el pragmatismo utilitarista y consumista, educando para el amor y la fraternidad hacia todas las personas y formas de vida con las que se convive en el planeta, desarrollo del pensamiento crítico con integridad y coherencia, generando espacios de indagación sobre el sentido de la vida; es decir, convertir la vida misma en objeto de búsqueda y conocimiento, se hace referencia así al "ser" esencial y más profundo, trascendiendo hacia lo que no se conoce como práctica de libertad, pero también de autodominio.

La propuesta educativa no podrá dejar de lado el humor, la alegría por la vida en contraposición a la parálisis vital; se trata de un sentir en el aula, pues la espiritualidad es una experiencia no racionalidad, una experiencia que se manifiesta en un quehacer de aula en felicidad, creatividad, libertad y deleite por la belleza de la realidad.

Se proponen a continuación algunas pautas para una práctica educativa bajo los principios de la espiritualidad:

- Reconocer los fundamentalismos y reduccionismos para proponer procesos educativos integradores, relacionales, resignificadores.

- Crear espacios didácticos, de modo que en los encuentros educativos se afirmen nuevas formas de pensar, ver y entender el mundo, los conocimientos y prácticas que en él se manifiestan.

- Propiciar relaciones humanas más profundas y trascendentes, comprendiendo que el acto educativo no puede entenderse únicamente en el presente de aula.

- Favorecer el diálogo abierto, la convivencia, la solidaridad, y la libertad con responsabilidad hacia toda forma de vida con la que se convive en el planeta.

- Apostar por la creatividad que emana de la libertad, la alegría y el juego.

La necesidad de un modelo educativo que atienda el desarrollo integral del ser humano y particularmente su esencia espiritual cobra nueva importancia frente al agotamiento de un sistema de desarrollo individual y colectivo económicamente injusto, y socialmente inhumano, brutal y alienado, tal y como lo afirma Maya (2013).

Estamos frente a nuevos tiempos, frente a una nueva sociedad con nuevos escenarios en todos los frentes de la actividad humana, frente a una ruptura de paradigmas de todo orden que han cumplido su misión o han descubierto que estaban equivocados a pesar de sus años de vigencia y la entronización de otros, frente a un ser humano que entiende sus desafíos a veces con angustia y otras afortunadamente con optimismo y esperanza. (p.276)

Por tanto, la esperanza del y para el cambio que impulse la reconstrucción de una ciudadanía planetaria y de un individuo en coherencia con su unidad compleja, está absolutamente ligada a una educación sustentada en lo espiritual que se manifiesta en un estado amoroso permanente de vocación y servicio, en la alegría y gozo por enseñar, en el compromiso social por la transformación y el cambio. Es una práctica educativa sustentada desde la espiritualidad, enseñando a vivir, propiciando el autodescubrimiento, el reconocimiento y el fortalecimiento de valores que dan sentido a la existencia.

\section{CONCLUSIONES}

La necesidad del desarrollo y fortalecimiento de la dimensión espiritual en la educación responde al momento de crisis que atraviesa el planeta, es un llamado a superar las visiones en las que se atienden 
únicamente las necesidades materiales y físicas que han dado por resultado conductas depredadoras en detrimento del otro y del planeta mismo.

El desarrollo de la espiritualidad presente en los seres humanos, posibilita la comprensión del ser, lo que al mismo tiempo promueve una vida con sentido y esperanza, apuesta por la felicidad, el respeto y la paz, no sólo consigo mismos, sino con todos y todo lo que les rodea, de este modo, se supera las visiones antropocentristas y se avanza hacia la construcción de una unidad planetaria y universal.

La educación se convierte en el agente por excelencia para el desarrollo de la espiritualidad, pero se trata de un modelo que en atención al paradigma educativo para el siglo XXI, comprenda y atienda un acto educativo en donde se asume una unión indivisible entre todas las dimensiones del ser humano y del propio conocimiento. Se trata de desarrollar prácticas educativas a través de la literatura, la música, el arte, la ciencia, y a la vez de fomentar creativamente el crecimiento de la dimensión espiritual, con el fin de lograr la comprensión de la integralidad y la relación del todo.

\section{REFERENCIAS}

Aguerrondo, I. (s.f.). El Nuevo Paradigma de la Educación para el siglo XXI. Recuperado de: http://www.oei. es/historico/administracion/aguerrondo.htm

Alves, R. (1976). Hijos del mañana. Ed. Sígueme, Salamanca.

Boff, L. (1992). Una espiritualidad liberadora. Ed. Verbo Divino, Navarra.

Boff, L. (2008). Saber cuidar. Ética do humano-compaixao pela terra. Petrópolis, RJ: Vozes. Rio de Janeiro.

Boff, L. (2013). El rescate de la categoría "espíritu". Recuperado de http://www.servicioskoinonia.org/boff/ articulo.php?num=544

Corbí, M. (2007). Hacia una espiritualidad laica: sin creencias, sin religiones, sin dioses. Ed. Herder, Barcelona.

Corbí, M. (2013). El silencio interior en una sociedad laica y global: estudio de las traducciones religiosas http://cetr.net/files/Silencio_Interior_en_la_Sociedad_Laica.pdf

Frank, V. (1987). Ante el vacío existencial. Ed. Herder Barcelona.

Gardner, H. (1983). Estructuras de la Mente. La Teoría de las Inteligencias Múltiples. Fondo de Cultura Económica, México.

Gómez, I. (2011). La competencia espiritual: Un proyecto para el futuro IX JORNADAS DE REFLEXIÓN SOBRE LA RELIGIÓN EN LA ESCUELA, Zaragoza en: http://www.deezaragoza.org/wp-content/ uploads/2011/02/Esquema.pdf

Guardans, T. Pérez, E. \& Robles, A. (2014). La educación para el desarrollo humano y la espiritualidad. Ed. UNIVERSITAS NUEVA CIVILIZACIÓN. Santiago.

Leme, M. (2014). Descubriendo caminos hacia una educación para la vida. UNA, Heredia.

Leme, M. (2015). Una pedagogía dialógica: hacia el desarrollo espiritual humano. Escuela Ecuménica de Ciencias de la Religión, Heredia.

Lugo, E. \& Barni, M. (2014). ¿Qué es la bioética? Vida y dignidad humana. Grupo Uno, Buenos Aires.

Maslow, A. (2008). La personalidad creadora. Ed. Kairós. Barcelona.

Maya, A. (2013). ¡Urgente! La educación debe cambiar. Transitando desde la educación tradicional a la educación holística. Coordinación Educativa y Cultural Centroamericana, San José. 
Ministerio de Educación Pública de Costa Rica (1994). Política educativa hacia el siglo XXI. Gobierno de Costa Rica, San José.

Morín, E. (1999). Los siete saberes necesarios para la educación del futuro UNESCO, París.

Morín, E. (2009). Introducción al pensamiento complejo. Ed. Gedisa, Barcelona.

Noblejas, M. (1994). Logoterapia. Fundamentos, principios y aplicación. Una experiencia de evaluación del "logro interior" Tesis Doctoral Universidad Complutense de Madrid Facultad de Educación.

Nogués, R. (2102). Emergencia evolutiva de la infraestructura de las experiencias de trascendencia. En Tragan, R. (Ed.) Neurociencias y espíritu. ¿Abiertos a una vida eterna? Ed. Verbo Divino.

Robles, A. (2011). Repensar la religión. De la Creencia al conocimiento. EUNA. Heredia.

Tedesco, J. (1995). El nuevo pacto educativo. Madrid: Grupo Anaya S.A. 